\title{
REVIEW \\ Adaptive Agricultural System to Dynamic Water Condition in a Low-Lying Area of Bangladesh
}

\author{
Haruo UCHIDA ${ }^{1 *}$ and Kazuo ANDO \\ Center for Southeast Asian Studies, Kyoto University (Kyoto, Kyoto 606-8501, Japan)
}

\begin{abstract}
The authors describe how farmers of Jawar village, which is located on the fringe of a large inundated area (haor) in Bangladesh, engage in agriculture and try to evaluate their traditional agricultural techniques. The farmers of the village cultivate a high yield variety (HYV) aman rice in high-elevation fields, a local variety (LV) in low-elevation fields and both varieties in medium-elevation fields to fit the ordinal water condition of the rainy season. In a flooding year they transplant LV aman rice even in parts of medium- and high-elevation fields where HYV is transplanted under ordinal circumstances in order to be well adapted to the dynamic hydrological condition. Aman rice cultivation in Jawar village is used to cope with flooding and water receding and the dry field crop (rabi) area increases in the following dry season to recover from losses in rice production of the former rainy season. Total rabi cultivated area of a flooding year is two times larger than that of the year before flooding, especially mustard cultivated area is three times larger. It can be said the farmers implement two countermeasures to increase crop production security; selecting a local rice variety in the rainy season and increasing rabi cropping area in the dry season have been adapted to meet the dynamic hydrological condition.
\end{abstract}

Discipline: Farming system

Additional key words: flood, kanda, rabi, traditional agriculture, transplanted aman

\section{Introduction}

It has been thought that a crop should be cultivated based on the deepest water level of each farmland in the rainy season of Bangladesh. This means flooding and planting are repeated on land classified by elevation in the same way every year. The fixed maximum water depth in the rainy season is the most important condition for agriculture in this farming system ${ }^{1}$, but the beginning and end of the rainy season, the amount of precipitation, the start of flooding and water recession, and the speed of these vary every year. If the absolute condition of maximum water depth is a static hydrological condition, the relative condition made by moving water could be called a "dynamic hydrological condition".

We tend to consider that a crop is selected to fit the fixed maximum water depth in traditional agriculture in the rainy season, but Bangladesh farmers positively select the crop variety to be cultivated and the cropping pattern based on the prediction of the dynamic hydrological condition. This fact has been neglected sometimes until now but is a fact of importance to evaluate the traditional agriculture in Bangladesh. In this paper, the authors describe how the farmers of Jawar village (Fig. 1) which is located on the fringe of a haor, a large inundated area in the rainy season that dries up in the dry season, engage in agriculture both in the rainy and dry seasons and try to evaluate the traditional agricultural techniques.

\section{Hydrological condition and land classification of Jawar village $^{2}$}

Fig. 2 shows the topography and land classification of Jawar village. The natural levee slopes towards the southeast in the middle of the village with a backswamp

This paper reports the results obtained in the joint project with the Government of Bangladesh on "Joint Study on Agricultural and Rural Development" during 1986 to 1989 sponsored by the Japan International Cooperation Agency.

Present address:

${ }^{1}$ Department of Hilly Land Research, Shikoku Research Center, NARCWR, NARO (Zentsuji, Kagawa 765-0053, Japan)

*Corresponding author: e-mail uchidah@affrc.go.jp

Received 20 June 2005; accepted 28 June 2006. 


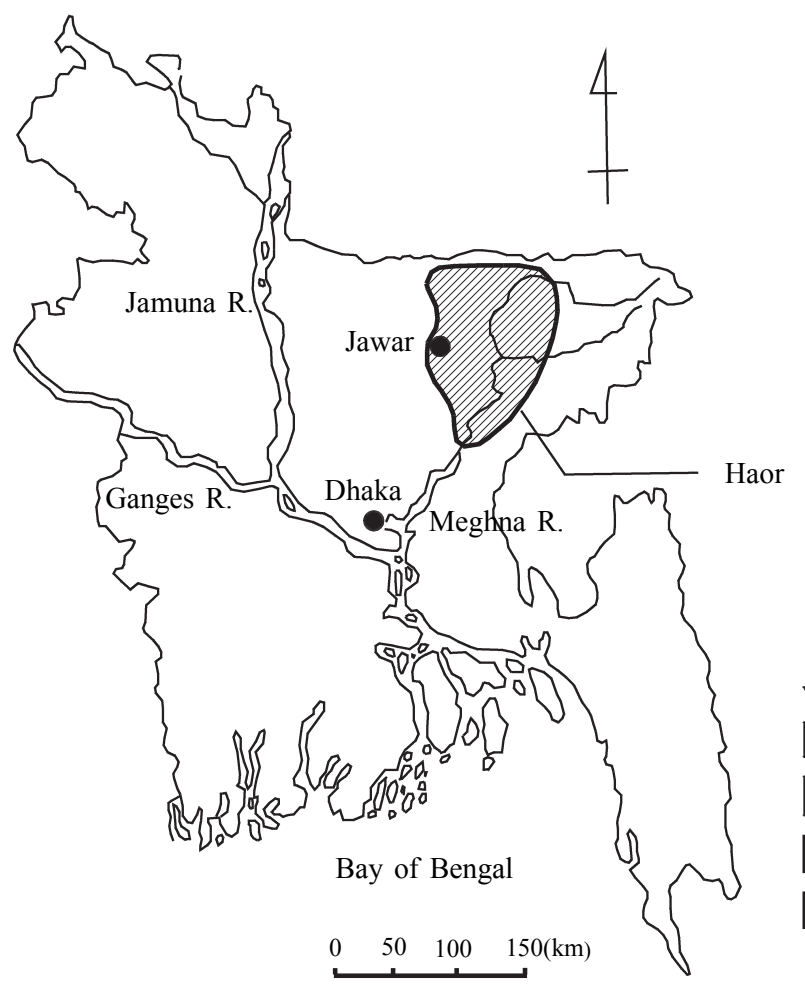

Fig. 1. Location of Jawar village

in the south which has some bils or marshes and a sloping floodplain that widely spreads in the north and east.

Hydrological conditions in both the rainy and the dry seasons vary with the topographical units. The rainy season starts from May or June and finishes in September or October. In the lowland areas the water level increases rapidly in May and reaches 5 to $7 \mathrm{~m}$ in the floodplain, and 2 to $3 \mathrm{~m}$ in the backswamp. Inundation disappears from the lowland in January or February. On the other hand, in the higher land on the natural levee the water level increases in July and recedes in October, and there is no inundation in the ordinal rainy season in the highest land including arable land of the west and homesteads.

Starting time, duration and depth of inundation vary in each topographical unit in the ordinal year and, under such hydrological condition, the farmers of the village classify the farmland into two basic types as shown in Fig. 2. One of these is the lowland called boro jami or shail jami which is located at 0 to $4 \mathrm{~m}$ elevation and another one is the highland called kanda at 4 to $7 \mathrm{~m}$ elevation.

It is impossible to cultivate in the rainy season on boro jami because of the excessive water depth and, on the other hand, in the dry season boro rice is cultivated using the abundant surface water. Boro jami which occupies half of the total land spreads to the backswamp and the lowland of the floodplain, most of which is used as boro rice single-crop farmland. Meanwhile, on kanda, it

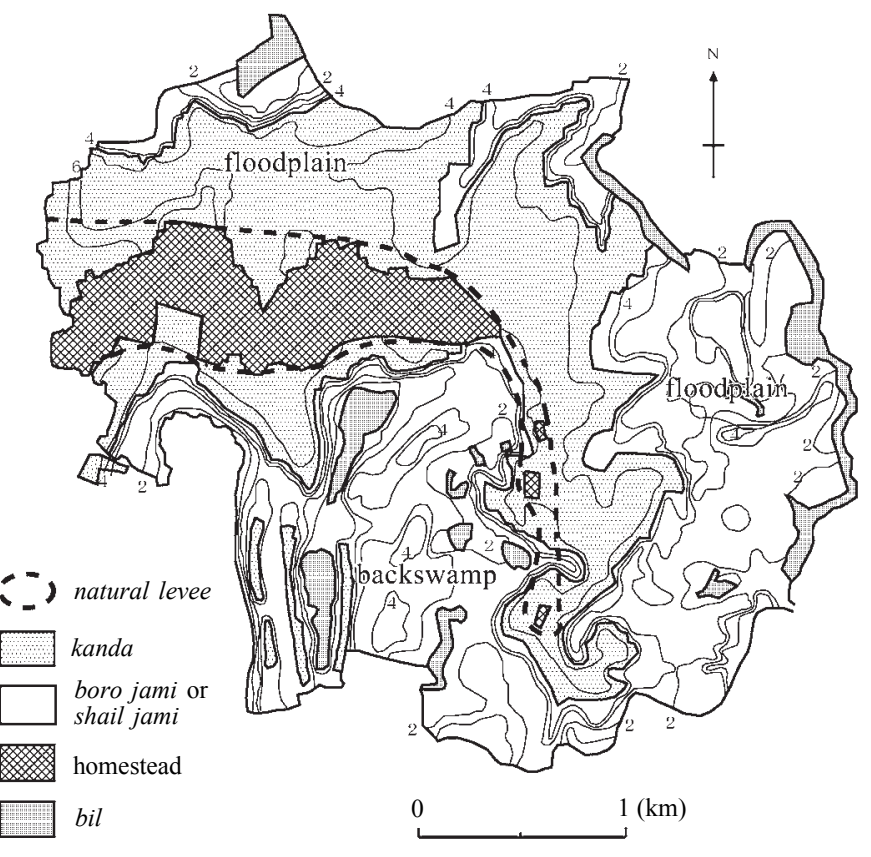

Fig. 2. Topography and land classification of Jawar village

is impossible to cultivate rice without irrigation in the dry season and the main rice cultivation on kanda depends on rainfall and inundation in the rainy season. Paddy fields on kanda are classified into high-, medium- and lowelevation fields and each field has a characteristic hydrological condition reflecting its elevation and topography respectively.

In Jawar village rice is cultivated at least once on $90 \%$ of the total farmland. In the dry season boro rice cultivation is dominant on the lowland area $(63.3 \%$ of total farmland) and, on kanda, the cropping patterns which include dry field crops (rabi) in the dry season based on aus of the early rainy season's rice and aman of the main rainy season's rice are dominant. The former and the latter patterns account for $4.6 \%$ and $22 \%$ of total farmland, respectively. The various cropping patterns on kanda mean the various hydrological conditions reflect its elevation and topography.

\section{Adaptive agricultural system of aman cultivation to dynamic hydrological condition}

\section{Rice variety and water-elevation condition}

Transplanted aman (T. aman) is cultivated on kanda intensively. Under the ordinal hydrological condition of the rainy season, namely in a non-flooded year, high yield variety (HYV) rice like pajam is mainly cultivated on the high-elevation fields, the local variety (LV) rice like mon- 
gir and/or biroi is cultivated on the low-elevation fields and both varieties are cultivated on the medium-elevation fields. Sixty-four percent of total T. aman cultivated in 1986 was HYV and most of these were pajam, and the other $36 \%$ was LV.

Agronomical characteristics of the rice variety and the water condition as a cultivating circumstance decide the variety planted in each field of the various elevations. The high-elevation fields are transplanted with HYV such as pajam at the beginning of August and ears form at the beginning of October when the rainy season is finishing. Transplanting time is fixed and the field is not inundated after transplanting in the ordinal year. Thus, intensive landuse is possible on the high-elevation fields throughout the year on the basis of stable and high yielding aman rice cultivation.

The young rice plants are transplanted when water starts to recede in the middle of August to the beginning of September on the medium-elevation fields and at the beginning of September to the beginning of October on the low-elevation fields with deep inundation of 30 to 50 $\mathrm{cm}$. Transplanting may be 2 weeks to a month later in the medium-elevation fields than the high-elevation fields and even one to two months later in the low-elevation fields if water recedes slowly. A local rice variety is mainly planted in the medium- and low-elevation fields. This kind of variety is more photosensitive than HYV and sensitively responds to day length, so the rice can be harvested at the end of November to the beginning of December even if rice stays in the field for a shortened period.

Thus, it is one of the characteristics of aman rice cultivation in Jawar village that HYV is cultivated in the high-elevation fields (yield of pajama in paddy is 4.6 $\mathrm{t} / \mathrm{ha}$ ), LV in the low-elevation fields (yield of mongir in paddy is $2.8 \mathrm{t} / \mathrm{ha}$ ) and both varieties are cultivated in the medium-elevation fields. It is notable that aman rice in the low-elevation fields where the water condition is very unstable is cultivated like rice transplanted at the water receding period of the dry season. Generally boro rice can not be cultivated in the rainy season because of deep inundation but can be transplanted after water starts to recede in the dry season. However in the village, a local rice variety is transplanted not only in the dry season, but also when water recedes after the peak of the flood even in the rainy season. We can understand this method of transplanting a local variety in a recognized low-elevation field as being remarkably one of the local and traditional techniques, which is adaptive to the water condition and the length of the cultivation period, both of which HYV cannot overcome because of its agronomical characteristics and moreover, this method is also efficient in avoiding flood damage.

\section{Selection of the rice variety for adaptation to the flood}

In the flooding year of 1987 , the local rice variety was transplanted even in parts of medium- and high-elevation fields where pajam rice had been planted in the ordinal year. Most of LV rice was transplanted after the second peak of flooding at and after the beginning of October because the farmers could not transplant HYV rice at a suitable time because of the deep inundation. In this case we can see the agricultural technique of transplanting when water recedes mentioned above.

The HYV which had occupied 64\% (76.8 ha) of total transplanted aman area in the former year decreased to $56 \%$ (22.4 ha) in this year and the LV increased to $44 \%$ (17.6 ha) from $36 \%$ (43.2 ha). The fact that the transplanted LV area increased in the flooding year in spite of a big reduction in total transplanted area means the farmers selected the transplanting variety as an adaptation to the flooding.

In Bangladesh, HYV rice has been considered to take the place of LV rice used in low-yielding traditional transplanted aman cultivation. Nevertheless, we can understand the traditional transplanting technique of LV when water recedes as a technique which is subtly adaptable to the dynamic hydrological condition neglected in HYV rice cultivation. The farmers of Jawar village are trying to recover from the damage of a flooding year by their traditional agricultural technique of using LV varieties, even though HYV is popular these days, in order to realize a stable transplanted aman cultivation.

\section{Adaptive agricultural system to dynamic water condition in rabi cultivation}

\section{Rabi cultivation in Jawar village}

The cultivated area of rabi crops in the village, namely upland crops in the dry season, is 110 ha $(13 \%$ of total farmland) in the $1986 / 87$ non-flood year. Most rabi crops like mustard, chili, wheat, and pulses are cultivated on kanda where the soil is rich in sand and drains well as shown in Fig. 3. These crops are sown from the beginning of November after reaping aman rice, in some cases the field is in fallow during the aman season, and are harvested in January and February.

In the village, rabi crops have been cultivated traditionally using water hyacinth brought by floods as manure and, in these days, the important cash crops are mustard and chili which increased in production after the construction of an embankment-cum-road to improve transportation in 1983. Especially, mustard which is cultivated widely and occupies $38 \%$ of total rabi cropped area $(1986 / 87)$ is the most important one. 


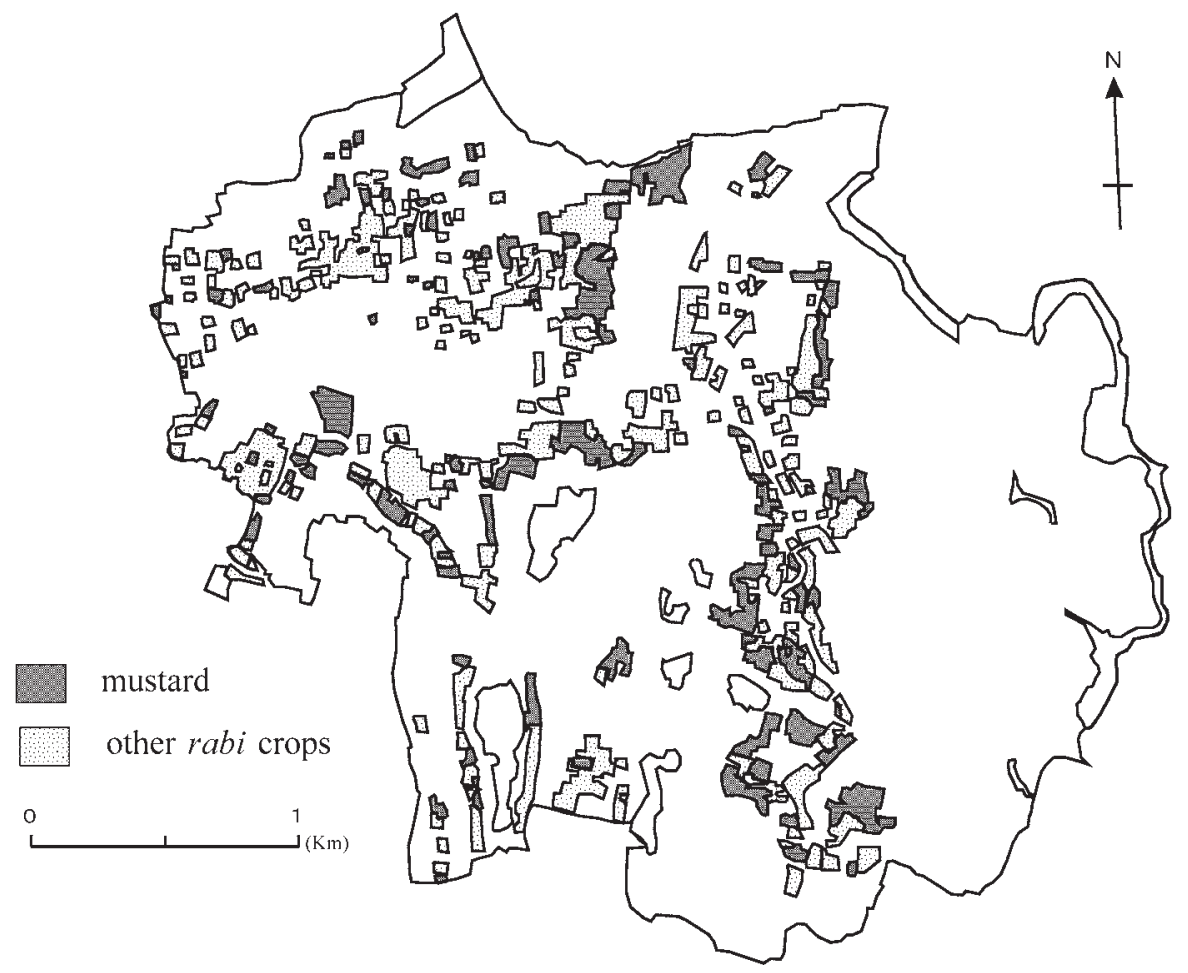

Fig. 3. Rabi crop cultivation in the non-flooding year (1986/87)

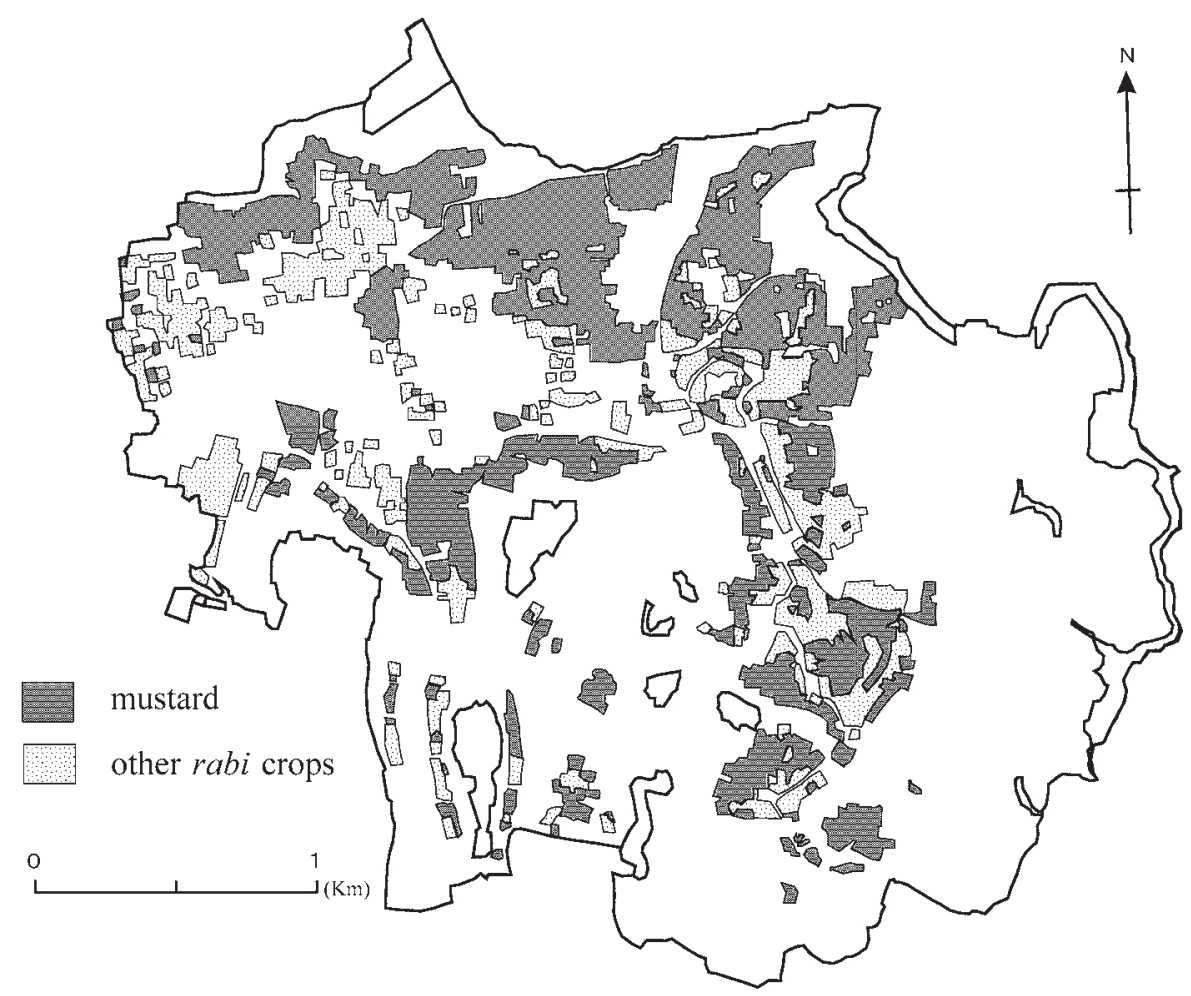

Fig. 4. Rabi crop cultivation in the flooding year (1987/88)

Plowing and soil shattering work for mustard cultivation starts in the middle or at the end of October when the moisture remaining in soil is still enough for cultiva- tion. Seeds are sown at the beginning of November after manuring and any fieldwork including tilling, weeding and irrigation is not needed until the harvest in February. 
Adaptive Agricultural System to Dynamic Water Condition in Bangladesh
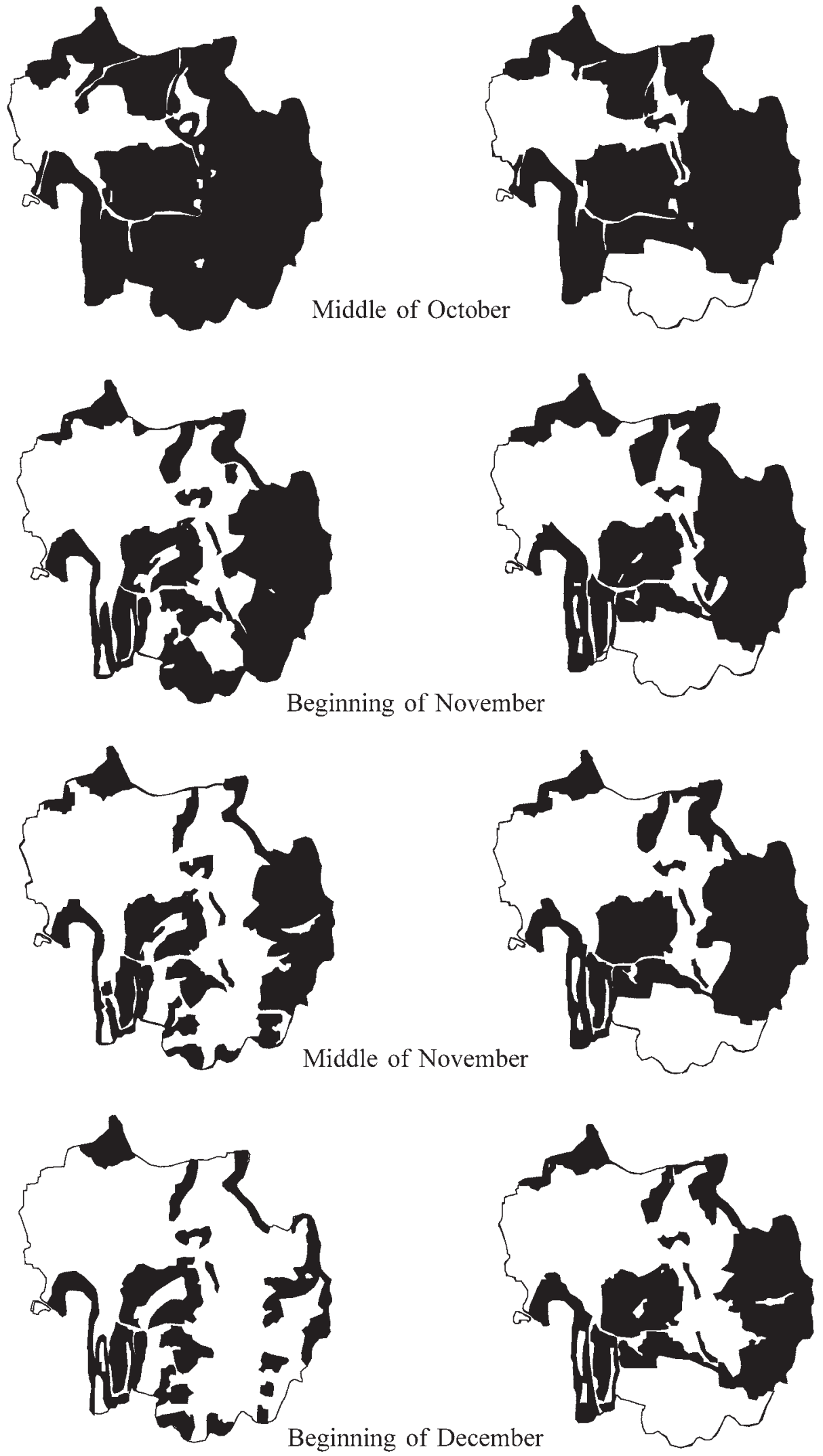

Flooding year (1987)

Non-flooding year (1986)

Fig. 5. Water recession in the flooding and non-flooding years

29 
Mustard cultivation takes the leading part in rabi cultivation because it requires much less labor than chili cultivation which requires irrigation many times.

\section{Adaptation to flood by rabi cultivation}

In Jawar village the rabi crop was cultivated on the medium- and low-elevation fields damaged by flooding after water had receded in the middle of November in 1987. Total rabi cultivated area was two times larger than the former year as shown in Table 1; mustard cultivated area was three times and chili area 1.8 times larger. The mustard cropped area was larger than other rabi crops and the ratio in rabi cropped area became 1.7 times larger (64\% of total rabi area).

As shown in Fig. 4, not only on the field of kanda which was given up to cultivate aman rice but also on the fallow field in the former year, rabi crop was newly cultivated and finally, in consequence, total cropped area became 10 ha larger than that of abandoned aman fields.

Fig. 5 shows the water recession patterns in 1986 and 1987. The southern area in 1986 is excluded because it was not researched. The inundated area in the middle of October in the flooding year of 1987 was larger than that of the former year and water receded rapidly afterwards. Especially, the water receding from kanda which is suitable land for rabi cultivation was remarkable at the beginning and the middle of November when mustard was sown. Thus, a great deal of water hyacinth carried by a large flood and the water that receded just in time resulted in abundant manure and moderate soil moisture good for rabi crops, especially mustard, in 1987/88.

The fact that the rabi cultivated area increases on a large scale after aman rice cultivation is damaged by flooding can be seen not only in Jawar village but in many other areas of Bangladesh. A bigger flood followed on the heels of the flood in 1987 and rice cultivation in the rainy season was damaged further in 1988, nevertheless, it is said the agricultural production nearly reached a record in $1988 / 89$. This shows that flood damage to agriculture in the rainy season can, on the contrary, guarantee some

Table 1. Rabi cropped area in the non-flooding and flooding years (ha)

\begin{tabular}{lcc}
\hline \hline Rabi crops & $\begin{array}{c}\text { Non-flooding year } \\
(1986 / 87)\end{array}$ & $\begin{array}{c}\text { Flooding year } \\
(1987 / 88)\end{array}$ \\
\hline Mustard & $41.8(38 \%)$ & $128.0(64 \%)$ \\
Chilli & $19.8(18 \%)$ & $32.2(16 \%)$ \\
Wheat & $26.4(24 \%)$ & $9.9(5 \%)$ \\
Pulses & $15.4(14 \%)$ & $23.7(12 \%)$ \\
Others & $6.6(6 \%)$ & $6.2(3 \%)$ \\
Total & $110.0(100 \%)$ & $200.0(100 \%)$ \\
\hline
\end{tabular}

crop cultivation conditions in the dry season. Namely, flooding in Bangladesh is a constraint for agriculture and, at the same time, is also a condition which can be used to maintain the balance of agricultural production.

\section{Conclusion}

The aman rice cultivation in Jawar village is coping with flooding and water receding and the rabi cropped area increases in the following dry season to recover the losses in rice production from the rainy season. It can be said the farmers implement two countermeasures to increase crop production security and adapt to the dynamic hydrological condition. We are apt to think that traditional agricultural techniques are passive but these techniques have been formed involving a positiveness which can be well adapted to the dynamic hydrological condition. This kind of positiveness or positive side of traditional agricultural techniques must be taken into consideration for flood control. It is very important how the dynamic hydrological condition is analyzed in addition to analyzing the static hydrological condition of the maximum depth of flood water in the rainy season and it is also important to find the traditional techniques adapted to the dynamic hydrological conditions. For this purpose researchers have to clarify the empirical knowledge of farmers for avoiding flood damages through interviews and observations.

For the improvement of agricultural techniques in Bangladesh, technical transfer from developed countries has been emphasized because of the high productivity of the transferred techniques. However, it is certain that new techniques, which have characteristics of the traditional agricultural techniques adapted to a changeable natural environment, must be developed and introduced. People taking part in rural development and aid to developing countries are required to recognize the rationality of traditional agriculture and should study "the existing local techniques" modestly.

\section{References}

1. Johnson, B. L. C (1982) Bangladesh (2nd ed.). Heinemann Educational Books, London, UK, 43.

2. Uchida, H. \& Ando, K. (1988) Tarail and Tangail villages. In Agricultural and rural development in Bangladesh, JSARD publication no. 6, eds. Khan, A. A., Hossain, S. M. A. \& Jaim, W. M. H., JICA, Dhaka, Bangladesh, 101136. 